\section{VARIATION IN ANTIBIOTIC USE IN NEONATAL INTENSIVE CARE UNITS IN THE NETHERLANDS}

\author{
Y. Liem ${ }^{1}$, T. Krediet ${ }^{2}$, A. Fleer ${ }^{3}$, T. Egberts ${ }^{1}$, \\ K. Rademaker ${ }^{1}$
}

${ }^{1}$ Clinical Pharmacy, ${ }^{2}$ Neonatology, University Medical Center Utrecht, Wilhelmina Children's Hospital, ' ${ }^{3}$ ijkman-Winkler Centre for Microbiology, Infectious Diseases and Inflammation, University Medical Center Utrecht, Utrecht, The Netherlands

Background and aims: Monitoring antibiotic use is essential to prevent overuse and misuse of antibiotics and to address the threat posed by resistant microorganisms. Studies have shown great variability in use of antibiotics between Dutch and European hospitals. Since empiric antibiotic therapy differs between NICUs and there is no consensus on the choice of antibiotics, we examined the variation in quantity and classes of antibiotics used in all ten tertiary care neonatal intensive care units (NICUs) in the Netherlands during 2005.

Methods: We collected data from all tertiary care NICUs in the Netherlands on clinical and demographic characteristics and the type and quantity of systemic antibiotic use (expressed as Defined Daily Doses (DDD)/100 admissions) in 2005. Antibiotics were ranked by volume of DDDs, and those antibiotics which accounted for $90 \%$ of the total volume of use (Drug Utilization (DU) $90 \%$ ) were noted. Treatment guidelines on antibiotic policy were investigated.

Results: Antibiotic consumption (based on pharmacy dispensing data) ranged from 130 to 360 DDD/100 admissions. In total 9-24 different antibiotics were used, of which 3-10 were in the DU90\%-segment.

The number of used antibiotics that were mentioned in the treatment guidelines ranged from $4(25 \%)$ to $10(77 \%)$ antibiotics.

Conclusions: By comparing antibiotic use in Dutch NICUs we found a considerable variation in the number of different antibiotics used and in the total amount of antibiotic use. Exploration of the opportunities to reach consensus in antibiotic policy, and to increase attention to antibiotic stewardship, is recommended.

\section{SCREENING OF INTERLEUKIN-1 RECEPTOR- ASSOCIATED KINASE 4 DEFICIENCY IN PATIENTS WITH HISTORY OF SEVERE PNEUMOCOCCAL INFECTIONS}

\author{
H. Yoshikawa' ${ }^{1}$, S. Watanabe ${ }^{2}$, H. Takada ${ }^{3}$
}

${ }^{1}$ Pediatrics, Nagaoka Habilitation Center for Severely Handicapped Children, Nagaoka, ${ }^{2}$ Neurology, Miyagi Children's Hospital, Sendai, ${ }^{3}$ Pediatrics, Graduate School of Medical Science, Kyushu University, Fukuoka, Japan

Introduction: Interleukin-1 receptor-associated kinase 4 (IRAK4) deficiency is animmunodeficiency disorder of the innate immune system resulting from inhibition ofToll-like receptor-mediated pathways. Some patients with IRAK4 deficiency experiencesevere bacterial infections until they reach three years of age; the infections weakenafter the age of four or five years. Therefore, the prevention of bacterial infection is apriority in the first three years of life for patients with IRAK4 deficiency. TNF-aproduction is almost completely inhibited in mononuclear cells after stimulation withlipopolysaccharide in IRAK4 deficiency. Consequently, TNF- $\alpha$ production can be usedas an early screen of IRAK4 deficiency in response to lipopolysaccharide in patientswith severe pneumococcal infection.

Method: Nine patients who had experienced severe pneumococcal meningitis or sepsis were included in this study. The three males and six females ranged from 7 months to 21 years. IRAK4 deficiency was assayed by measuring the TNF- $\alpha$ production of mononuclear cells using flow cytometry following the stimulation of peripheral blood cells with lipopolysaccharide (J. Pediatr 2006; 148:546).

Result: All nine patients showed normal TNF- $\alpha$ production (84.8 - 97.4\%).

Conclusion: None of the patients exhibited IRAK4 deficiency. However, due to thesmall number of subjects, further study using this screening method with a largernumber of participants may detect latent IRAK4 deficiency in patients. 